\title{
Chip-on-tip endoscope incorporating a soft robotic pneumatic bending microactuator
}

\author{
Benjamin Gorissen $^{1 *}$, Michael De Volder ${ }^{1,2}$, Dominiek Reynaerts ${ }^{1}$ \\ ${ }^{1}$ Department of Mechanical Engineering, KU Leuven and Flanders Make, Celestijnenlaan 300, 3001 Leuven, \\ Belgium \\ ${ }^{2}$ Institute for Manufacturing, Department of Engineering, University of Cambridge, 17 Charles Babbage Road, \\ Cambridge CB3 OFS, UK \\ * corresponding author: benjamin.gorissen@ kuleuven.be, +3216322758
}

\begin{abstract}
In the ever advancing field of minimally invasive surgery, flexible instruments with local degrees of freedom are needed to navigate through the intricate topologies of the human body. Although cable or concentric tube driven solutions have proven their merits in this field, they are inadequate for realizing small bending radii and suffer from friction, which is detrimental when automation is envisioned. Soft robotic actuators with locally actuated degrees of freedom are foreseen to fill in this void, where elastic inflatable actuators are very promising due to their S3-principle, being Small, Soft and Safe. This paper reports on the characterization of a chip-on-tip endoscope, consisting out of a soft robotic pneumatic bending microactuator equipped with a $1.1 \times 1.1 \mathrm{~mm}^{2} \mathrm{CMOS}$ camera. As such, the total diameter of the endoscope measures $1.66 \mathrm{~mm}$. To show the feasibility of using this system in a surgical environment, a preliminary test on an eye mock-up is conducted.
\end{abstract}

Keywords Minimally invasive surgery, Chip-on-tip endoscope, Bending actuator, Soft robotic actuator

\section{Introduction}

During the last decades, surgeons endeavored to reduce the invasiveness of procedures. Reducing the surgical trauma drastically led to a shortening of hospitalization with an overall reduction in post-surgical complications (Fuchs, 2002). The introduction of minimally invasive surgery (MIS), where instruments are introduced into the body through millimeter-sized keyholes, pushed technology to develop ever smaller tools without a loss of functionality. The quest for hightech surgical tools became even more stringent when surgeons started to use natural orifices as access points to the internal body (NOTES). As such, there is a vast medical need for small surgical instruments that are able to navigate through the human body, that are inherently safe but still have functional end effectors.

Soft robotic actuators are especially interesting to be used in tools for MIS and NOTES. In contrast to conventional robotic systems, where typically materials are used with a Young's modulus of $10^{9}-$ $10^{12} \mathrm{~Pa}$, soft robots are made out of materials with moduli in the same range as biological soft tissue $\left(10^{4}\right.$ $-10^{9} \mathrm{~Pa}$ ) (Rus et al., 2015). The advantage of using soft actuator materials is that, when in contact with biological tissue, the soft actuator cannot impose detrimental stresses. As such, safety limits do not have to be imposed by software algorithms, but they are inherently present in the hardware of the robot. For small scale soft robotic actuators, a number of actuation principles are possible: electrically, magnetically, chemically, thermally (including SMA), photo responsive and pressure driven (Hines et al., 2017). Especially pressure driven actuators are interesting for MIS and NOTES procedures since they avoid the use of high electric or magnetic fields and do not contain high temperature parts (De Greef et al., 2009). Although systems under pressure also carry an inherent risk, the pressures used in elastic inflatable actuators (EIAs) are usually low $(<300 \mathrm{kPa})$, and when saline water is used as a pressure medium, fluid leakages impose no thread to the surrounding tissue (Okayasu et al., 2005). Further, several studies have shown that at microscale, the highest force and power densities are delivered by hydraulic and pneumatic actuators (De Volder and Reynaerts, 2010).

Earliest reports on EIAs date back to 1919 (Fleury, 1919), however a big breakthrough came with the work of Suzumori et al. (Suzumori, 1989) in 1989 that made a mm-scaled bending inflatable actuator. Since then 
EIAs flourished in literature and surgical applications were increasingly envisioned. Konishi et al. reported on a cuff actuator for adaptive holding around nerves (Konishi et al., 2002), a pair of surgical forceps that are pneumatically actuated (Fujiwara et al., 2009) and bending retractors in front of endoscope for submucosal dissection (Konishi, 2011). Haga et al. (2005) developed a hydraulic active bending catheter, a 2 DOF bending actuator was utilized in semiautonomous colonoscopy by Chen et al. (2009) and Devreker et al. (2014) envisioned to use McKibben actuators to steer a robotic catheter for trans-catheter aortic valve implantations. A general surgical manipulator is envisioned by the company Materialise NV (Schiettecatte, 2014) and by Ciancetti et al. (2013) in the Stiff-flop project, that incorporates a central stiffening channel. A more exhaustive list on EIAs in a surgical context can be found in (De Greef et al., 2009; Hines et al., 2017).

In this paper, a soft robotic actuator pressurized by air is used that shows a bending deformation when inflated. This actuator essentially consists out of a cylindrical central void that is asymmetrically placed in a flexible cylinder, as is shown in Fig. 1. When taking a longitudinal cross-section of the actuator structure, it can be seen that the pressure void is enclosed by a thin wall on one side and a thick wall to the other side. When inflated, the structure will bend towards the thick-walled side. This is in contrast to planar designs where a rectangular void is placed asymmetrically in a rectangular outer structure, where a bidirectional motion can be seen (Gorissen et al., 2011; Gorissen et al., 2011), which is less desirable in a surgical context.

As will we demonstrated in this paper, the bending actuator is augmented with a CMOS camera to create a chip-on-tip endoscope. This shows the potential of micro-EIAs in the domain of MIS and NOTES, where it could be used to visualize parts of the body that are hard to access with current technology. An example of such a procedure is shown on Fig. 2, where a brain ventricle is visually inspected using the foreseen endoscope. Commercial flexible endoscopes generally consist of a central glass fiber, that guides light from top to base of the endoscope. This fiber is then bend by means of pulling on eccentrically placed cables. The central fiber restricts the minimal attainable bending radius, and is omitted in the chip-on-tip concept. Here, electrical signals need to be conveyed from endoscope tip to its base by means of electrical leads. When flexible electronics are used, the minimally attainable bending radii can be drastically reduced.

This paper will go into detail on the manufacturing and characterization of both a single bending actuator and a one degree-of-freedom (DOF) bending pneumatic endoscope. Further, its application in a mock-up surgical procedure will be demonstrated.

\section{Manufacturing}

\subsection{Single bending actuator}

The elastic bending actuator is produced using a onestep micromolding process, as can be seen on Fig. 3. It is made out of polydimethylsiloxane (PDMS), a rubber that is known for its biocompatibility (Ratner et al., 2004). In general, inflatable actuators out of silicone rubbers can suffer from a sudden large expansion of the cross-section due to a small pressure increment (ballooning) (Elsayed et al., 2014), which can be solved by including strain limiting fibers (Konishi et al., 2005). However, previous research (Gorissen et al., 2011) showed that actuators out of PDMS behave more predictably and do not require these fibers. For producing the cylindrical actuators in this research, both halves of the mold are micromilled out of aluminum, where the bottom part of the mold is fitted with a tungsten carbide microrod $(\varnothing 0.6 \mathrm{~mm})$ that forms the internal inflatable void of the actuator. The top halve of the mold comprises a $\varnothing 1 \mathrm{~mm}$ drilled hole to shape the outside of the actuator. A precise alignment by means of dowel pins of both halves of the mold is needed to ensure an eccentricity of $0.14 \mathrm{~mm}$ between inner void and outer cylinder. Prior to molding, both halves of the mold are coated with a demolding agent (liquid release agent, Devcon), which is needed for high aspect-ratio demolding. PDMS (Sylgard 184, Dow Corning) is mixed in a 10:1 ratio and degassed in vacuum. After filling and closing of the mold, it is put on a hot plate $\left(70^{\circ} \mathrm{C}\right)$ for 1 hour, to ensure full crosslinking of the silicone rubber. The hollow cylindrical actuator is finished after demolding and trimming the excess rubber at the bottom by means of a scalpel.

\subsection{Chip-on-tip endoscope}

The flexible fluidic bending actuator is combined with a small digital camera to form a chip-on-tip endoscope. The used CMOS camera (NanEye, Awaiba) has a footprint of $1.1 \times 1.1 \mathrm{~mm}^{2}$ with a resolution of $250 \times 250$ pixels, where each pixel measures $3 \times 3 \mu \mathrm{m}^{2}$. The sensors is fitted with an optical lens, having a field of view of $90^{\circ}$ in air, $62^{\circ}$ in water and a depth of focus between 3.5 and $30 \mathrm{~mm}$, meaning that the objects to be registered needs to lay within this distance from the lens.

An assembly of this flexible endoscope is shown on Fig. 4. The bending actuator is glued to a hollow cylindrical brass tube with inner diameter of $1 \mathrm{~mm}$ and outer diameter $1.5 \mathrm{~mm}$ that serves both as a rigid holder and as a pressure supply. To connect the camera to the bending actuator, a camera holder is 3D-printed out of titanium using selective laser melting. This holder, with an outside diameter of $1.66 \mathrm{~mm}$ is designed to conformally hold the camera while it is glued to the bending actuator on the other side. The outside diameter of the holder is the largest cross-sectional dimension of the endoscope, and is the limiting 
dimension for keyhole applications. By avoiding the camera holder and directly embedding the camera in the flexible actuator during the molding process, these dimensions can be further reduced. As such, the hard limit of this process is the size of the camera.

\section{Characterization}

\subsection{Static}

The static deformation of only the pneumatic actuator is registered using a digital microscope at different pressure inputs and is depicted on Fig. 5. At each input pressure level, the actuator shape forms a circle segment, where at $250 \mathrm{kPa}$ a bending deformation of $160^{\circ}$ (inscribed angle of the circle segment) can be seen. The same test is done for the assembled endoscope with results shown on Fig. 6. When loaded at a pressure of $280 \mathrm{kPa}$, a bending deformation of $45^{\circ}$ of the actuator can be seen. Compared to the previous static test results of the free bending actuator, this stroke reduction is due to the ribbon cable that is attached to the camera. The ribbon cable consists out of 4 silver plated copper electrical leads with diameter of $71 \mu \mathrm{m}$ and a Young's modulus of about $120 \mathrm{GPa}$. The resulting bending stiffness is much higher than that of the PDMS bending actuator (Young's modulus of $500 \mathrm{kPa}$ ) alone, causing the stroke reduction.

\subsection{Dynamic}

The dynamic performance of a single, free bending actuator is assessed by mean of a high-speed camera test setup. To include the effect of a possible wet environment the endoscope has to work in, dynamic tests are conducted in both air and water. The bending actuator is placed in a transparent PMMA container which can be filled with water and loaded with a pressure step of $200 \mathrm{kPa}$. The pressure step is imposed using a fast switching ( $<2 \mathrm{~ms}$ switching time) solenoid valve (MHE2, Festo). As will be shown, the valve is an order of magnitude faster than the actuator, such that the measured response can be considered independent of the valve dynamics. Further, the distance between valve, actuator and pressure transducer is minimized ( $<10 \mathrm{~mm}$ ), to ensure minimal dynamic losses in effect of the supply lines. High-speed camera pictures were taken using a CMOS camera (HighSpeedStar 5, LAVision) at a frame rate of $1500 \mathrm{~Hz}$. From these images, the end point of the bending actuator is tracked, resulting in the step response (SR) curve in Fig. 7. On this figure the relative position of the tip is plotted along its actuation path, with a value of ' 1 ' indicating a full stroke, and this in air (solid line) and in water (hatched line).

From these step response curves and assuming that the system is a first order system, the time constant $(\tau)$ of the actuator can be deduced. This is the time that the actuator takes to complete $63 \%$ of its full stroke. In air this time constant is measured to be $11 \mathrm{~ms}$ while in water this is $14 \mathrm{~ms}$. This can be related to the bandwidth of the actuator $(\mathrm{BW}=1 /(2 \pi \tau))$, to be $14 \mathrm{~Hz}$ in air and $11 \mathrm{~Hz}$ in water. The reduction of bandwidth in water in relation to in air is caused by viscous drag forces of water on the actuator, which act as a damper on the system. In relation to normal surgical procedures where the surgeons movements are almost entirely below 2 $\mathrm{Hz}$ (Hu et al., 2006), the bandwidth of the bending actuator is well sufficient for surgical tasks. Especially when taking into account that the dynamic requirements of visualization tools are far less stringent than for manipulation instruments.

\section{Application}

To show the advantage of using elastic inflatable microactuators in a surgical context, the flexible chipon-tip bending endoscope is used in an mock-up ophthalmological procedure where the retina is to be visualized. An overview of the procedure can be seen on Fig. 8, or as a movie in the supplementary materials. The inset images labeled as "endoscopic view", show the images captured by the NanEye camera at the tip. In this test, the flexible endoscope is inserted through the pupil of the eye, instead of through an incision in the sclera. For easy access, cornea and lens are removed beforehand. After insertion but before actuation (middle image, top row), the camera only captures a limited area of the retina around the blind spot (lighter colored spot from witch a retinal vein is protruding). After imposing a pressure input of $280 \mathrm{kPa}$ (right image, bottom row), it can be seen that another section of retina become visible. By a consequent depressurizing of the actuator, the endoscope returns to its initial state, without noticeable hysteresis, as was also reported in (Gorissen et al., 2011). Although in this procedure, only one side of the retina was visualized, an expansion to $360^{\circ}$ can be implemented by rotating the endoscope around the axis of the pressure holder, which would accord to a rotation inside the surgical trocar.

\section{Discussion}

As can be seen by the static bending deformation of the endoscope (Fig. 6) and by the mock-up procedure (Fig. 8) the signal transferring cable of the camera has a negative impact on the system performance. The large discrepancy between cable stiffness and actuator stiffness lies at the origin of this. A great potential is foreseen in the use of flexible electronics to fully incorporate flexible leads in the actuator design. In this context, the reader is referred to (Kim et al., 2011) where on a balloon catheter conductive leads are patterned to form sensors for measuring temperature, flow, tactile, optical and electrophysiological data, 
together with radiofrequency electrodes for ablation of tissue. However on a small scale, flexible electronics necessitate the use of lithographical techniques to produce the inflatable bending actuator. Therefore the circular design of the actuators needs to be substituted for a planar design with rectangular cross section, that that can be made using soft-lithography techniques (Gorissen et al., 2011), or using normal lithography (Gorissen et al. , 2016).

The concept of using a CMOS or CCD camera at the tip of an endoscopic tool has already been commercialized in flexible cable driven endoscopes with HD image registration (Tan and Preminger, 2004). This paper showed the feasibility of using a chip-on-tip concept in a surgical context by incorporating a soft robotic inflatable bending actuator. Compared to a cable or concentric tube driven approach (Webster and Jones, 2010), inflatable bending actuators have that advantage that the minimal attainable bending radius can be made smaller, making it possible to navigate through more intricate parts of the body where sharp corners need to be made without imposing detrimental forces to the environment. Further, while it is difficult to induce other deformations beside translation and bending by pulling on cables or pushing on rubes, EAIs in literature have been developed that, besides bending, are able to generate all other fundamental deformation modes: contraction (Takemura et al., 2005), extension (Martinez et al., 2012) and torsion (Gorissen et al., 2014). Since they are all operated by increasing the induced pressure, they can be easily combined in series to form a complex surgical soft robot adapted to the environment of the envisioned procedure. However, pressure transfer still remains an issue for a serial soft robot: supplying each actuator with its own tubing will rapidly become too bulky, while intermediate active valves are very hard to downscale into the submillimeter range (Moers et al., 2012). A possible solution consists of embedding hardware intelligence in the soft robotic systems, where external boundaries can trigger sequential motion (Glozman et al., 2010). Alternatively, an on-board pressure generation system can also be used to avoid pressure supply lines. An on board pressure generation would have the added advantage that the dynamics of the systems are not compromised by long pressure supply lines. Although fully passively controlled systems have been reported in literature (Wehner et al., 2016), inflatable untethered servo-controlled slave systems, operated by a human master still remains a topic to be explored in the domain of surgical soft robotic systems.

The materials used in inflatable soft robotics by definition have a low Young's modulus, leading to large attainable deformations at relatively low pressure loadings. As such, these actuators are foreseen to play an important role in applications where only low actuation forces can be exerted, as is precisely the case in endoscopic procedures. When larger forces are needed, solutions in literature have been suggested to locally change the stiffness of the actuator (Cianchetti et al., 2013). However these solutions are only viable if external forces need to be resisted, not if they need to be exerted. A further issue with inflatable soft robotic actuators is that although they can be made inherently safe, they are susceptible to mechanical damage by sharp objects, like being punctured by a needle or cut by a blade. Inevitably, this will lead to an inability to build up pressure with a resulting loss in functionality. Even though damaged actuators will remain safe and back-drivable, inflatable materials with low Young's modulus have been developed and incorporated in flexible actuators that are more resilient to mechanical damage (Martinez et al., 2014) or are even self-healing (Terryn et al., 2017). Lastly, the used materials in inflatable soft robotics all exhibit a strong non-linear stress-strain relation. This eventually leads to a nonlinear input-output behavior of the actuator. This leads to unintuitive actuator behavior, where the same pressure increment results in a different bending increment in the beginning of the stroke versus at the end of the stroke. To cope with this drawback, actuator performance can be made more linear by altering the actuator design (Mosadegh et al., 2014) without a loss in actuator performance.

\section{Conclusion}

This paper reports on a new type of flexible endoscope, by combining a chip-on-tip CMOS camera with an elastic inflatable microactuator with bending deformation. The actuator is made by PDMS micromolding that produces the actuator in one step, without the need of bonding or manual alignment. The actuator, with outer diameter of $1 \mathrm{~mm}$ and length 10 $\mathrm{mm}$, is able to bend $160^{\circ}$ at a pressure loading of 250 $\mathrm{kPa}$ and exhibits a bandwidth of $14 \mathrm{~Hz}$ in air and $11 \mathrm{~Hz}$ in water. Consequently, this actuator is incorporated in a flexible endoscope, using a manual assembly process, where a static test shows a bending deformation of $45^{\circ}$ at $280 \mathrm{kPa}$. This reduction in performance is attributed to the signal transferring cable that is attached to the used CMOS sensor. Globally, the used endoscopic sensors measures 1.66 millimeter in diameter and 12 millimeter in length. To show the feasibility of using the flexible endoscope in a surgical setting, a surgical procedure was simulated were the retina of a mock-up eye was visualized using the developed technology.

Acknowledgements This research is supported by the Fund for Scientific Research-Flanders (FWO), and the European Research Council (ERC starting grant HIENA). 


\section{References}

Chen, G., Pham, M. T., \& Redarce, T. (2009). Sensor-based guidance control of a continuum robot for a semiautonomous colonoscopy. Robotics and Autonomous Systems, 57(6-7), 712-722.

Cianchetti, M., Ranzani, T., Gerboni, G., De Falco, I., Laschi, C., \& Menciassi, A. (2013, 2013

Nov 03-08). STIFF-FLOP Surgical Manipulator: mechanical design and experimental characterization of the single module. Paper presented at the IEEE/RSJ International Conference on Intelligent Robots and Systems (IROS), Tokyo, JAPAN.

De Greef, A., Lambert, P., \& Delchambre, A. (2009). Towards flexible medical instruments: Review of flexible fluidic actuators. Precision Engineering-Journal of the International Societies for Precision Engineering and Nanotechnology, 33(4), 311-321.

De Volder, M., \& Reynaerts, D. (2010). Pneumatic and hydraulic microactuators: a review. Journal of Micromechanics and Microengineering, 20(4).

Devreker, A., Vander Poorten, E., Gijbels, A., Tran, P. T., De Pratere, H., Herijgers, P., et al. (2014). Towards fluidic actuation for catheter-based interventions. Paper presented at the Proceedings Actuator 2014.

Elsayed, Y., Vincensi, A., Lekakou, C., Geng, T., Saaj, C. M., Ranzani, T., et al. (2014). Finite Element Analysis and Design Optimization of a Pneumatically Actuating Silicone Module for Robotic Surgery Applications. Soft Robotics, 1(4), 255-262.

Fleury, L. A. (1919). US1295471.

Fuchs, K. H. (2002). Minimally Invasive Surgery. Endoscopy, 34(02), 154-159.

Fujiwara, N., Sawano, S., \& Konishi, S. (2009). Linear expansion and contraction of paired pneumatic balloon bending actuators toward telescopic motion. 2009 IEEE 22nd International Conference on Micro Electro Mechanical Systems. MEMS 2009, 435-438.

Glozman, D., Hassidov, N., Senesh, M., \& Shoham, M. (2010). A Self-Propelled Inflatable Earthworm-Like Endoscope Actuated by Single Supply Line. Ieee Transactions on Biomedical Engineering, 57(6), 1264-1272.

Gorissen, B., Chishiro, T., Shimomura, S., Reynaerts, D., De Volder, M., \& Konishi, S. (2014). Flexible pneumatic twisting actuators and their application to tilting micromirrors. Sensors and Actuators a-Physical, 216, 426-431.

Gorissen, B., De Volder, M., De Greef, A., \& Reynaerts, D. (2011). Theoretical and experimental analysis of pneumatic balloon microactuators. Sensors and Actuators aPhysical, 168(1), 58-65.

Gorissen, B., Donose, R., Reynaerts, D., \& De Volder, M. (2011). Flexible pneumatic micro-actuators: analysis and production. Procedia Engineering, 25, 681-684.

Gorissen, B., Van Hoof, C., Reynaerts, D., \& De Volder, M. (2016). SU8 etch mask for patterning PDMS and its application to flexible fluidic microactuators. Microsystems \& nanoengineering, 2.

Haga, Y., Muyari, Y., Mineta, T., Matsunaga, T., Akahori, H., Esashi, M., et al. (2005). Small diameter hydraulic active bending catheter using laser processed super elastic alloy and silicone rubber tube. Paper presented at the 3rd IEEE/EMBS Special Topic Conference on Microtechnology in Medicine and Biology, Oahu, HI.

Hines, L., Petersen, K., Lum, G. Z., \& Sitti, M. (2017). Soft Actuators for Small-Scale Robotics. Advanced Materials, 29(13).
Hu, J., Chang, C.-Y., Tardella, N., Pratt, J., \& English, J. (2006). Effectiveness of haptic feedback in open surgery simulation and training systems. Stud. Health Technol. Inform, 119, 213-218.

Kim, D.-H., Lu, N., Ghaffari, R., Kim, Y.-S., Lee, S. P., Xu, L., et al. (2011). Materials for multifunctional balloon catheters with capabilities in cardiac electrophysiological mapping and ablation therapy. [10.1038/nmat2971]. Nat Mater, 10(4), 316-323.

Konishi, S. (2011). Small, soft, safe micromachine for minimally invasive surgery. 2011 International Meeting for Future of Electron Devices (IMFEDK), 20-21.

Konishi, S., Kobayashi, T., Maeda, H., Asajima, S., \& Makikawa, M. (2002). Cuff actuator for adaptive holding condition around nerves. Sensors and Actuators B-Chemical, 83(13), 60-66.

Konishi, S., Nokata, M., Jeong, O. C., Sakakibara, T., Kusuda, S., Kuwayama, M., et al. (2005). Merging micro and macro robotics toward micro manipulation for biomedical operation. Paper presented at the The 36th International Symposium on Robotics.

Martinez, R. V., Fish, C. R., Chen, X., \& Whitesides, G. M. (2012). Elastomeric Origami: Programmable Paper-Elastomer Composites as Pneumatic Actuators. Advanced Functional Materials, 22(7), 1376-1384.

Martinez, R. V., Glavan, A. C., Keplinger, C., Oyetibo, A. I., \& Whitesides, G. M. (2014). Soft Actuators and Robots that Are Resistant to Mechanical Damage. Advanced Functional Materials, 24(20), 3003-3010.

Moers, A. J. M., De Volder, M. F. L., \& Reynaerts, D. (2012). Integrated high pressure microhydraulic actuation and control for surgical instruments. Biomedical Microdevices, 14(4), 699-708.

Mosadegh, B., Polygerinos, P., Keplinger, C., Wennstedt, S., Shepherd, R. F., Gupta, U., et al. (2014). Pneumatic Networks for Soft Robotics that Actuate Rapidly. Advanced Functional Materials, 24(15), 2163-2170.

Okayasu, H., Okamoto, J., Fujie, M. G., \& Ieee. (2005). Development of a hydraulically-driven flexible manipulator including passive safety method. Paper presented at the IEEE International Conference on Robotics and Automation (ICRA), Barcelona, SPAIN.

Ratner, B. D., Hoffman, A. S., Schoen, F. J., \& Lemons, J. E. (2004). Biomaterials Science: An Introduction to Materials in Medicine: Elsevier Science.

Rus, D., \& Tolley, M. T. (2015). Design, fabrication and control of soft robots. Nature, 521(7553), 467-475.

Schiettecatte, P., \& Plaghki, R. (2014). WO2014131810A1.

Suzumori, K. (1989). Flexible Microactuator. Trans. of Japan Society of Mechanical engineers, 55(518), 2547-2552.

Takemura, K., Yokota, S., \& Edamura, K. (2005, 18-22 April 2005). A Micro Artificial Muscle Actuator using Electroconjugate Fluid. Paper presented at the Robotics and Automation, 2005. ICRA 2005. Proceedings of the 2005 IEEE International Conference on.

Tan, Y. H., \& Preminger, G. M. (2004). Advances in video and imaging in ureteroscopy. Urologic Clinics of North America, 31(1), 33-42.

Terryn, S., Brancart, J., Lefeber, D., Van Assche, G., \& Vanderborght, B. (2017). Self-healing soft pneumatic robots. Science Robotics, 2(9).

Webster, R. J., \& Jones, B. A. (2010). Design and Kinematic Modeling of Constant Curvature Continuum Robots: A Review. International Journal of Robotics Research, 29(13), 1661-1683.

Wehner, M., Truby, R. L., Fitzgerald, D. J., Mosadegh, B., Whitesides, G. M., Lewis, J. A., et al. (2016). An integrated design and fabrication strategy for entirely soft, autonomous robots. Nature, 536(7617), 451-455. 


\section{Figures}

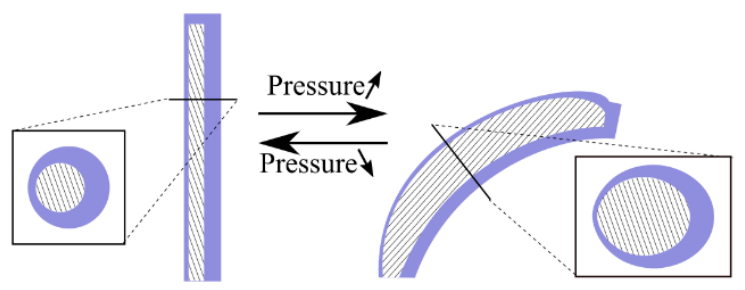

Fig. 1 Schematic overview of the bending deformation of an elastic inflatable microactuator.
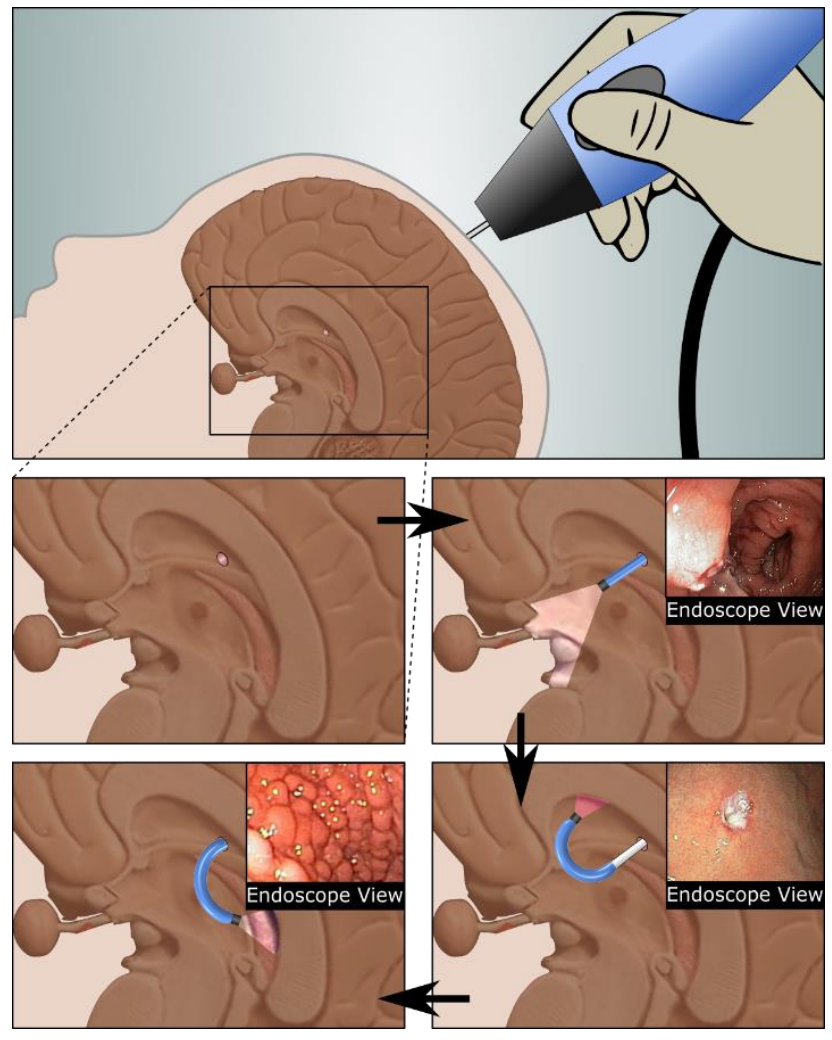

Fig. 2 Minimally invasive surgical procedure where a chip-on-tip endoscope is used to visualize a brain ventricle. An elastic inflatable bending actuator is used to increase the field of view of the endoscope. 


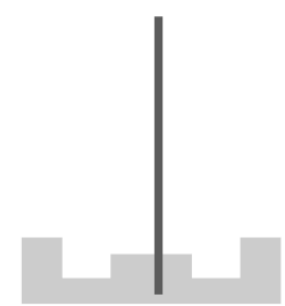

a) $\mu$-milling of mold and $\mu$-rod insertion

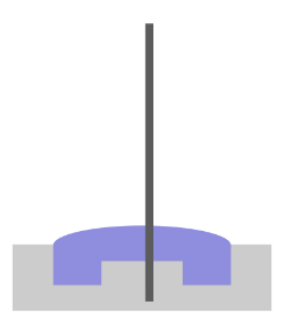

b) Filling of mold with PDMS

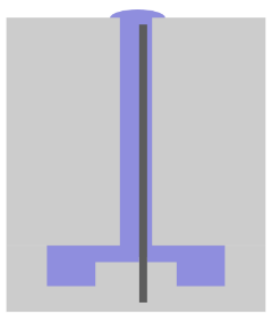

c) Closing of mold (overflow of PDMS on top)

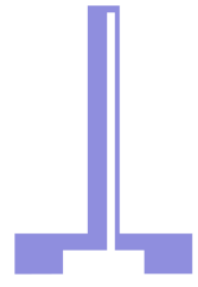

d) Demolding of actuator

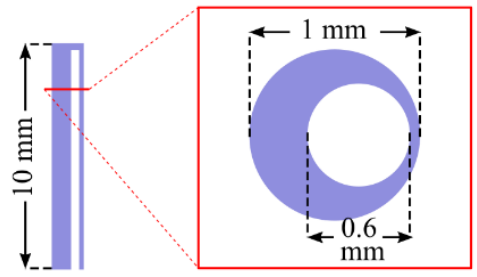

e) Trimming of excess

Fig. 3 Overview of the production process for an elastic inflatable microactuator with bending deformation.

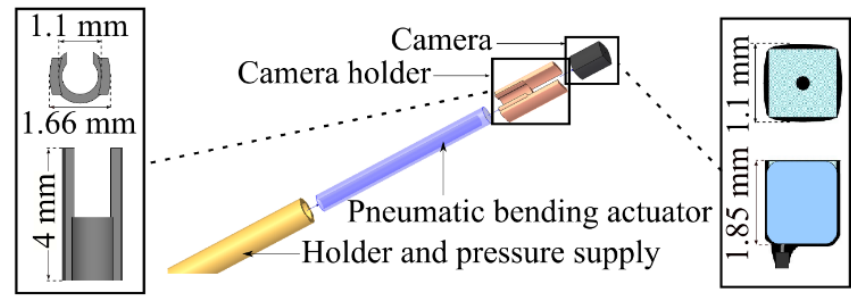

Fig. 4 Assembly of a flexible endoscope with chip-on-tip camera using an elastic inflatable microactuator with bending deformation.

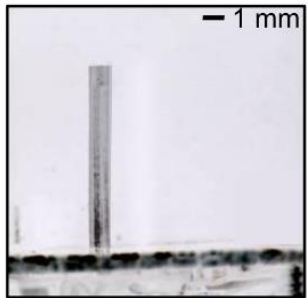

$0 \mathrm{kPa}$

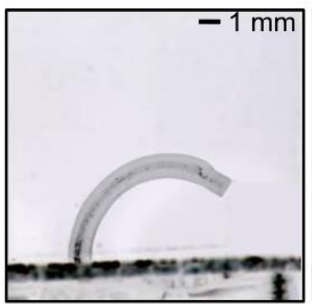

$200 \mathrm{kPa}$



$100 \mathrm{kPa}$

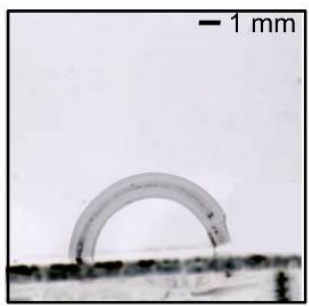

$250 \mathrm{kPa}$

Fig. 5 Static deformation of a pneumatic bending microactuator with outer diameter $1 \mathrm{~mm}$, inner diameter $0.6 \mathrm{~mm}$, eccentricity $0.14 \mathrm{~mm}$ and length $10 \mathrm{~mm}$. 

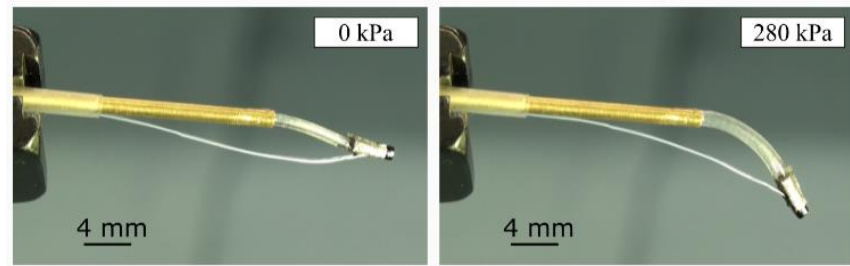

Fig. 6 Static deformation of a flexible endoscope with chip-on-tip CMOS camera before and after loading at a pressure of $280 \mathrm{kPa}$.

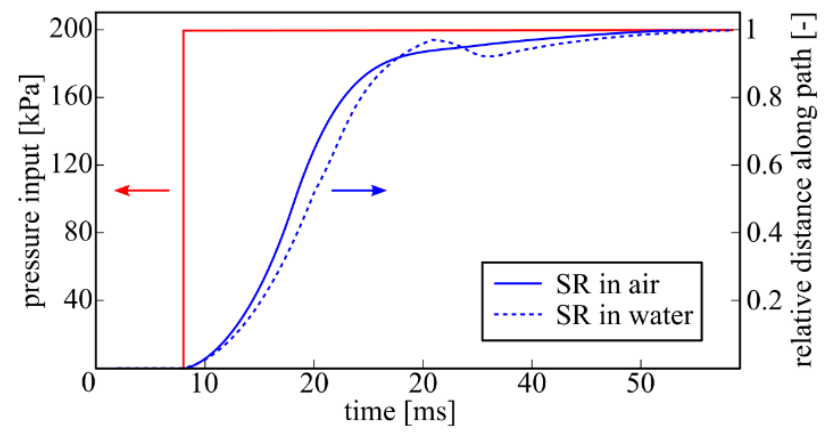

Fig. 7 Step response (SR) of a pneumatic bending actuator with outer diameter of $1 \mathrm{~mm}$, length of $10 \mathrm{~mm}$, inner diameter of $0.6 \mathrm{~mm}$ and eccentricity of $0.14 \mathrm{~mm}$, in air (solid line) and water (hatched line).
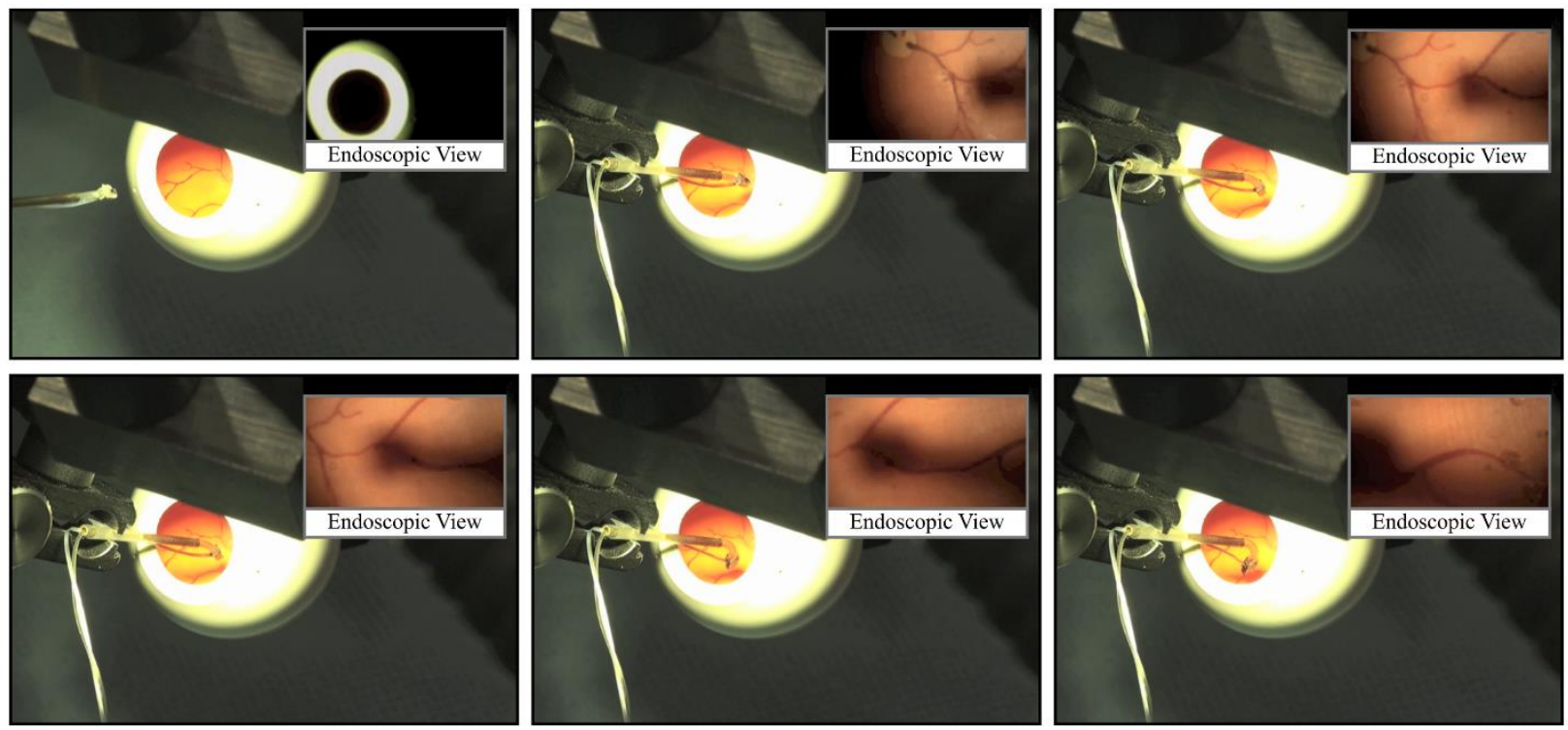

Fig. 8 Mock-up ophthalmological procedure where the retina of an eye is visualized using the developed chip-ontip endoscope using an elastic inflatable actuator with a one DOF bending deformation. Sequence of images is from left to right and from top to bottom, which are taken from the movie in supplementary information. 